\title{
Aspectos Biométricos y Morfológicos del Pericardio Fibroso y Diafragma en el Hombre
}

\author{
Biometric and Morphological Charachteristics of the \\ Fibrous Pericardium and the Diaphragm in Man
}

"Octavio Binvignat $\&$ **Enrique Olave

BINVIGNAT, O.\& OLAVE, E. Aspectos biométricos y morfológicos del pericardio fibroso y diafragma en el Hombre. Int. J. Morphol., 27(3):841-847, 2009.

RESUMEN: El pericardio es una membrana fibro-serosa que envuelve al corazón y a la porción yuxtacardíaca de los grandes vasos. Realizamos un estudio del pericardio y del diafragma, registrando sus dimensiones, sus relaciones, así como también, establecer el tipo de conexiones existente entre ambas estructuras. Fueron disecadas 142 regiones mediastínicas de cadáveres sin fijación o con fijación en formaldehído al 10\%, brasileños, adultos, de ambos sexos, de edades comprendidas entre los 18 y 70 años, fallecidos de diferentes causas. Para el estudio histológico, del conjunto pericardio y diafragma fueron retirados cinco fragmentos de diferentes regiones: anterior próxima al esternón (región 1), lateral izquierda próxima al ápice del corazón (región 2), posterior (región 3 ), lateral derecha próxima al paso de la vena cava inferior (región 4) y central (región 5). El promedio de los diámetros latero-lateral y antero-posterior del pericardio fueron de $103,3 \pm 6,7$ y $66,0 \pm 2,3 \mathrm{~mm}$, respectivamente y del diafragma de $309,4 \pm 27,4$ y $152,5 \pm 24,9 \mathrm{~mm}$, respectivamente. El área del diafragma fue en promedio de $37.260 \pm 2.324 \mathrm{~mm}^{2}$. El área de la base del pericardio sobre el diafragma fue de $6.042 \pm 367$ $\mathrm{mm}^{2}$. El espesor del diafragma fue en promedio: parte derecha, 2,42 $\pm 0,34 \mathrm{~mm}$; parte izquierda, 2,38 $\pm 0,71 \mathrm{~mm}$ y la parte anterior, 2,52 $\pm 0,66 \mathrm{~mm}$. El promedio del espesor del pericardio separado del diafragma fue de 0,26 $\pm 0,02 \mathrm{~mm}$. En la región 2 ambas estructuras fueron separadas con facilidad en 47,2 mm; en la región 5 ambas estructuras se encuentran fusionadas. Los resultados obtenidos en este trabajo complementarán los conocimientos morfológicos sobre el pericardio fibroso y sus relaciones con el diafragma.

PALABRAS CLAVE: Anatomía; Diafragma; Morfología; Biometría.

\section{INTRODUCCION}

El pericardio es una membrana fibro-serosa que envuelve al corazón y a la porción yuxtacardíaca de los grandes vasos. Esta envoltura se encuentra firmemente adherida al tendón central del diafragma, perdiéndose a nivel de las vainas perivasculares en la base del corazón. Con el progreso de la cirugía cardiovascular y con el desafío técnico de las revascularizaciones cardíacas, se han incrementado las investigaciones sobre el trayecto de los vasos para dichas intervenciones, por lo que se hace necesario tener claro las características de las envolturas del corazón y su relaciones. La literatura muestra que varios autores han citado la anatomía del pericardio y su relación con el diafragma (Wallraff, 1937; Debrünner, 1956; DiDio, 1985; Latremouille et al., 1999 , entre otros), pero se debe complementar sobre su morfología y parámetros biométricos.
Basado en lo anterior, nos propusimos realizar un estudio sistemático del pericardio y del diafragma en la parte en que se contactan, registrando sus dimensiones, sus relaciones, así como también, establecer el tipo de conexiones existente entre ambas estructuras.

\section{MATERIAL Y MÉTODO}

Para el presente estudio, fueron disecadas 142 regiones mediastínicas de cadáveres sin fijación o con fijación en formaldehído al 10\%, de individuos brasileños, adultos, de ambos sexos, de edades comprendidas entre los 18 y 70 años, fallecidos por diferentes causas. Los cadáveres estaban lo-

\footnotetext{
* Universidad Autónoma de Chile, Sede Talca, Chile.

** Facultad de Medicina, Universidad de La Frontera, Chile.
} 
calizados en la Escuela Paulista de Medicina y en el Centro Anatómico de la Universidad de Alfenas e Instituto Médico Legal de Alfenas, Minas Gerais, Brasil.

Los cadáveres fueron colocados en decúbito dorsal, realizando incisiones que permitieran la visualización de los elementos en estudio. Se retiró el peto esternocostal y los órganos torácicos. La cavidad pericárdica fue abierta por su parte anterior, retirando el corazón, para así tener una visión del área pericárdica relacionada al diafragma. Posteriormente, registramos las siguientes medidas:

1. Pericardio diafragmático:

1.1.Dimensión transversal (mayor diámetro transverso).

1.2.Dimensión antero-posterior (margen anterior al margen posterior, punto medio).

1.3.Dimensiones de espesor.

\section{Diafragma:}

2.1.Dimensión transversal (Parte más lateral del diafragma)

2.2.Dimensión antero-posterior (inserción esternal e inserción lumbar).

2.3.Dimensiones del espesor.

Las mediciones de la base del pericardio fueron obtenidas configurando un triángulo dentro del espacio ocupado por éste en el diafragma, donde se midieron sus lados y la distancia entre éstos y los márgenes del espacio.

Las disecciones fueron realizadas con el auxilio de una lupa estereoscópica. Las mediciones fueron registradas con un caliper digital marca Mitutoyo, de precisión $0,05 \mathrm{~mm}$.

Para la parte histológica, del conjunto pericardio y diafragma fueron retirados cinco fragmentos de diferentes regiones: región anterior próxima al esternón (región 1), región lateral izquierda próxima al ápice del corazón (región 2), región posterior (región 3), región lateral derecha próxima al paso de la vena cava inferior (región 4) y región central (región 5). Todos los fragmentos fueron tratados con técnicas histológicas de rutina.

Los fragmentos retirados tenían la forma de un triángulo para reconocer los planos y dirección de las estructuras, marcados y colocados en recipientes plásticos individuales para su posterior estudio microscópico.

En 24 piezas provenientes de cadáveres no fijados, se realizó técnica de disección mesoscópica, dejándolas en una solución de vinagre comercial (ácido acético) durante 48 horas a una temperatura de $35^{\circ} \mathrm{C}$ en estufa convencional.

\section{RESULTADOS}

Descripción biométrica. Los datos correspondientes a los promedios de los diámetros latero-lateral y antero-posterior del pericardio en el plano horizontal y del diafragma desinsertado y extendido sobre una superficie plana son mostrados en la Tabla I. Ambas estructuras se observan claramente en las Figuras 1 y 2.

\begin{tabular}{lccc}
\multicolumn{4}{l}{ Tabla I. Diámetros promedio del pericardio y diafragma $(\mathrm{mm})}$. \\
\hline Diámetros & $\mathrm{n}$ & Promedio & D. S. \\
\hline latero-lateral pericardio & 142 & 103,3 & 6,7 \\
antero-posterior pericardio & 142 & 66,0 & 2,3 \\
latero-lateral diafragma & 142 & 309,4 & 27,4 \\
antero-posterior diafragma & 142 & 152,5 & 24,9 \\
\hline
\end{tabular}

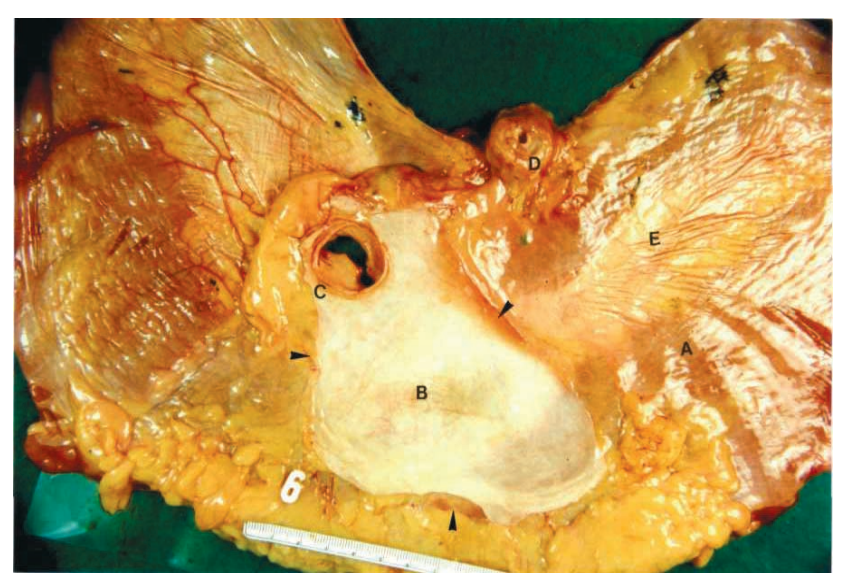

Fig. 1. Diafragma desinsertado (A) y pericardio (B) de un hombre adulto, 47 años, colocado en una superficie plana. Se observa el límite irregular del pericardio (puntas de flecha), foramen de la vena cava (C) de forma circular, esófago (D), hojuela izquierda del tendón central del diafragma (E).

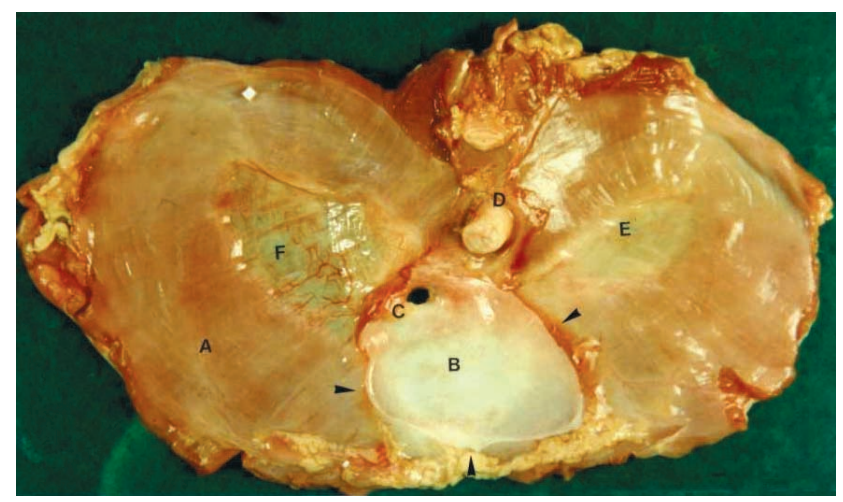

Fig. 2. Diafragma desinsertado (A) y pericardio (B) de un cadáver de sexo femenino, 18 años, colocado en una superficie plana. Se observa que el pericardio presenta una forma de triángulo con márgenes curvilíneas (punta de flechas). Foramen de la vena cava inferior (C) de forma circular, esófago (D), hojuela izquierda (E) y hojuela derecha $(\mathrm{F})$ del tendón central del diafragma. 
De acuerdo a los resultados, el área del diafragma tuvo un promedio de $37.260 \pm 2.324 \mathrm{~mm}^{2}$.

De las medidas obtenidas, tanto en pericardio como en diafragma y realizando una comparación entre sexos, no se encontraron diferencias estadísticamente significativas.

Con respecto al triángulo trazado dentro del espacio ocupado por el pericardio en el diafragma, la longitud del lado derecho del triángulo tuvo un promedio de 66,4 \pm $2,98 \mathrm{~mm}$; el lado anterior tuvo $91,1 \pm 3,44 \mathrm{~mm}$ y en el lado posterior (que sería la hipotenusa) se registró 118,0 \pm 5,67 $\mathrm{mm}$.

La distancia entre el lado anterior del triángulo y el margen anterior del espacio ocupado por el pericardio en el diafragma fue en promedio $6,0 \pm 1,37 \mathrm{~mm}$; entre el lado derecho del triángulo y el margen derecho del espacio en el diafragma fue de 7,0 $\pm 3,2 \mathrm{~mm}$ y entre el lado posterior del triángulo y el margen posterior del espacio fue de 12,0 $+3,45 \mathrm{~mm}$.

Los valores obtenidos permitieron calcular el área ocupada por el pericardio en el diafragma, primeramente como un trígono dentro de ese espacio y luego sus áreas adyacentes. De acuerdo a los resultados obtenidos el área ocupada por el pericardio en el diafragma fue de $6.042 \pm$ $367 \mathrm{~mm}^{2}$.

En relación al espesor del diafragma, los promedios obtenidos fueron: parte derecha, $2,42 \pm 0,34 \mathrm{~mm}$; parte izquierda, 2,38 $\pm 0,71 \mathrm{~mm}$ y la parte anterior, 2,52 $\pm 0,66$ $\mathrm{mm}$. El promedio del espesor del pericardio separado del diafragma fue de $0,26 \pm 0,02 \mathrm{~mm}$

Descripción Histológica. El análisis histológico de los tejidos que participan en la conformación del bloque pericardio-diafragmático en sus diferentes regiones presentó las siguientes características:

Región 1. El pericardio está representado por un tejido conjuntivo denso unido a la porción membranosa del diafragma, con tejido adiposo interpuesto. Se pudo observar la transición del tejido conjuntivo denso del diafragma tendinoso para el tejido muscular del mismo.

Entre el pericardio y el tejido tendinoso y muscular del diafragma se encuentra tejido adiposo (Fig. 3)

Región 2. En esta región se observa contacto del pericardio con el diafragma. El pericardio es más delgado en esta porción y se encuentra representado por tejido conjuntivo denso. Entre ambas estructuras hay presencia de tejido adiposo.

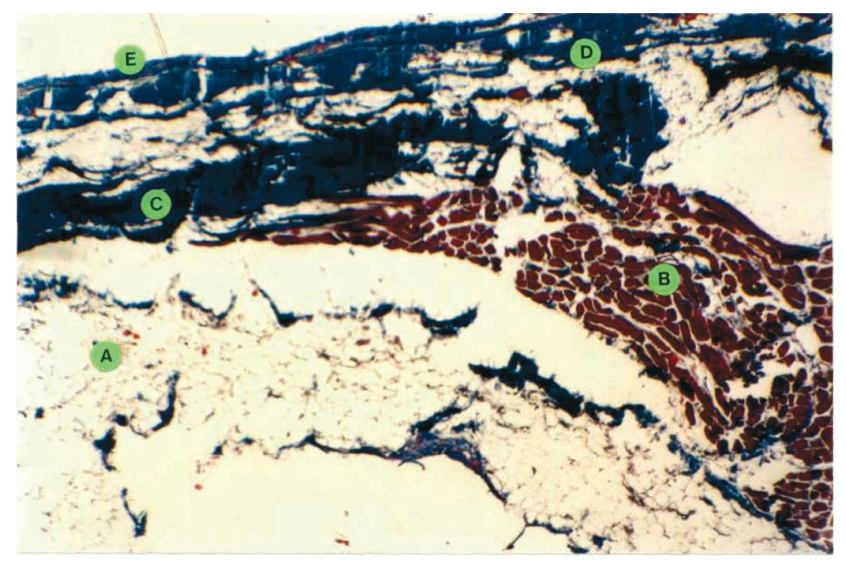

Fig 3. Microfotografía mostrando parte del diafragma y pericardio humanos correspondientes a la región 1 . Se observa tejido adiposo (A), tejido muscular diafragmático(B) y tejido conjuntivo $(\mathrm{C}=$ tendón central del diafragma; $\mathrm{D}=$ pericardio parietal y $\mathrm{E}=$ fascia endotorácica). Entre el pericardio y el tendón central, notar infiltrado de tejido adiposo. Tricrómico de Masson. 270 X.

Región 3. Aquí el pericardio se encuentra formado por tejido conjuntivo denso, cuyas fibras se entrecruzan en la porción en contacto con el diafragma, el cual presenta gran concentración de tejido adiposo.

Región 4. El pericardio está representado por tejido conjuntivo rico en fibras colágenas orientadas en varios sentidos. Entre el pericardio y el diafragma se observa tejido adiposo. La parte más externa del pericardio presenta fibras separadas por tejido adiposo.

Región 5. El diafragma está constituído básicamente por tejido conjuntivo denso, con fibras colágenas que se entrecruzan en el plano transversal (Fig. 4).

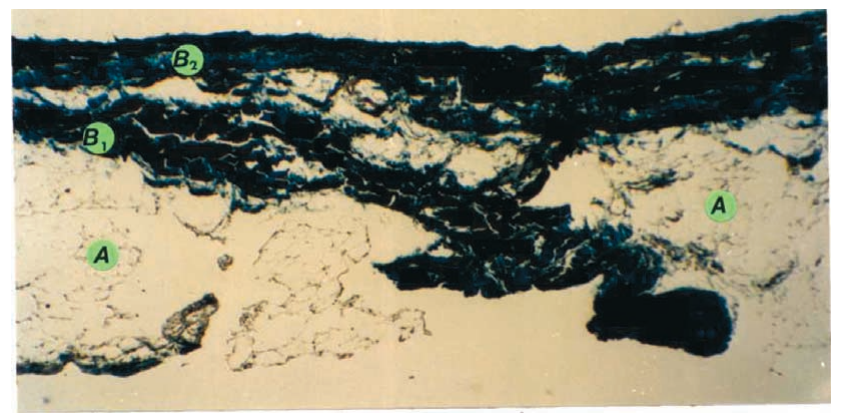

Fig. 4. Microfotografía mostrando parte del diafragma y pericardio correspondiente a la región 5. Observar tejido adiposo (A) del peritoneo, tejido conjuntivo denso (B1 y B2) parte del tendón central del diafragma (B1) y pericardio fibroso (B2). Tricrómico de Masson. $360 \mathrm{X}$.

\section{Relaciones pericardio -diafragmáticas}

Región 1. En la parte anterior, los limites de la relación 
pericardio-diafragmática no son bien definidos debido a la infiltración de tejido adiposo y también por la presencia de los ligamentos esterno-pericárdicos inferiores. En dirección posterior, el tejido conjuntivo es más denso, uniendo el pericardio al diafragma, lo que dificulta la separación de las dos estructuras.

Región 2. En esta parte hay un tejido conjuntivo laxo transparente, fino, con fibras paralelas que se continua con la fascia endotorácica. Al disecar esas fibras, el pericardio continuaba adherido por un tejido conjuntivo laxo con infiltraciones de tejido adiposo, siendo fácil de desprender hasta 47, $2 \mathrm{~mm}$ de su margen posterior, pasando frente al esófago y a la hojuela media del tendón central. Próximo a la hojuela derecha, la disección fue difícil, ya que las fibras se entrecruzaban formando una unidad con el diafragma, lo que ocurrió a 26,7 mm del margen posterior.

Región 3. La parte posterior presenta las mismas características de la región precedente.

Región 4. En el lado derecho, la disección fue facilitada hasta 12,3 $\mathrm{mm}$ de su margen derecho. Cuando el pericardio entra en contacto con la parte fibrosa del diafragma, las fibras se entrecruzan. Del mismo modo que en el lado opuesto, observamos las siguientes capas: un tejido liso transparente con fibras ordenadas recubriendo al diafragma y continuándose con la fascia endotorácica; un tejido conjuntivo laxo con infiltraciones de tejido adiposo, interpuesto entre el pericardio y el diafragma; fibras formando un ángulo agudo en la base del pericardio, las que se entrecruzan con fibras del diafragma.

Región 5. La parte central se encuentra firmemente adherida al diafragma por el entrecruzamiento de las fibras que forman una unidad, pero es también la parte de menos espesor del bloque pericardio-diafragma.

\section{DISCUSIÓN}

El pericardio es una membrana fibro-serosa que envuelve al corazón y las partes adyacentes de sus grandes vasos. En general, la literatura no se refiere en forma detallada a parámetros biométricos de las relaciones entre el pericardio y el diafragma. Sin embargo, hay variados reportes sobre hernias pericardio-diafragmáticas (Larrieu et al., 1980), rupturas pericardio-diafragmáticas (Sharma,1999), agenesia parcial (Scapellato et al., 1996) o congénita total del pericardio (Abbas et al., 2005), lo que sin duda, hace de estas estructuras un tema de mucho interés y de la necesidad de profundizar los conocimientos sobre ellas.
La literatura clásica de anatomía refiere descripciones similares y en relación a la parte del pericardio que esta formando su base en el diafragma, Poirier \& Charpy (1902); Gérard (1912); Fusari (1913); Fumagalli \& Cavallotti (1982), describieron que tenía una forma triangular u oval semejante a las observadas en nuestra serie. Sin embargo, varios autores como Fort (1902); Poirier \& Charpy; Berdal (1906); Tillaux (1908); Gérard; Davis (1913); Landouzi \& Bernard (1921); Chiarugi (1924b); Le Gross Clark (1949); Maisonnet \& Coudane (1950); Bruce et al. (1964); Orts Llorca (1972); Lockhart et al. (1972); Prives et al. (1984); Wilson \& Wilson; Bouchet \& Cuilleret (1979); Basmajian (1980) y Moore (1990) describieron al pericardio como un cono achatado antero-posteriormente con una base elíptica.

Los promedios obtenidos en nuestras muestras (103 $\mathrm{mm}$ para diámetro transversal y $66 \mathrm{~mm}$ para antero-posterior) es concordante con los informes de Valenti (1909); Versari (1932); Maisonnet \& Coudane; Bouchet \& Cuilleret que citaron que la base del pericardio en el diafragma tenía $10 \mathrm{~cm}$ transversalmente y $7 \mathrm{~cm}$ anteroposteriormente ; Paturet (1958) y Testut \& Latarjet (1969) describieron de 9 a $11 \mathrm{~cm}$ transversalmente y 5 a $6 \mathrm{~cm}$ en sentido anteroposterior. Por su parte Fumagalli \& Cavallotti indicaron que el diámetro transversal corresponde a 13 o $14 \mathrm{~cm}$, valor bastante más alto que los mencionados previamente.

Un aspecto interesante fue relatado por Versari y Gérard, resultado que también fue observado en nuestras disecciones. El área del triángulo no corresponde exactamente a la base, ya que el volumen pericárdico es un poco más amplio que ella, motivo por el cual existe un pliegue pequeño no adherente, limitado por un surco a la izquierda donde se inserta la pleura parietal, originándose así un receso freno-pericárdico, pero en el lado derecho esta descripción no es tan clara.

De acuerdo a los resultados obtenidos en esta investigación, el área del pericardio en su base relacionada con el área del diafragma, tiene una proporción de 1: 6 .

Con relación a la adherencia o fusión del pericardio al diafragma, las descripciones de Anile (1919); Leeson \& Leeson (1972); Romanes (1976); Snell (1981) y Moore han señalado que el pericardio se une a la porción tendinosa y/o muscular del diafragma, de forma adherente o fusionada, sin embargo, en nuestras muestras, en la región 2 es posible separar estas estructuras por 47,2 $\mathrm{mm}$ aproximadamente, mientras que en las regiones 1 y 3 son de difícil separación; en la 4 se permite una pequeña separación periférica y ya en la 5, existe un entrecruzamiento de fibras en varios sentidos, lo que no permite separar ambas estructuras. Descripciones de Williams et al. (1995) señalan que en algunos mamíferos 
inferiores, la base está completamente separada del diafragma o unida solamente por tejido areolar. Estos mismos autores citaron que la inserción diafragmática en el ser humano es tejido fibroareolar laxo, fácilmente separable a excepción de una pequeña área del tendón central donde se fusiona con el pericardio.

Las observaciones en nuestro material han mostrado que la zona de adherencia se encuentra en el margen anterior del triángulo y en la parte central del mismo, diferente de lo expresado por Gérard que describió a ésta reduciéndose en sentido antero-posterior después de pasar de la mitad, lo que corresponde a la unión del $1 / 3$ anterior con los $2 / 3$ posteriores de la distancia entre la columna vertebral y el esternón.

El pericardio relacionado con el diafragma debe ser dividido en dos zonas: la primera, unida al diafragma por diferentes tipos de tejidos, conjuntivo laxo y denso y, la segunda, zona libre, marginal de $1,0 \mathrm{~cm}$ rodeando a la anterior en casi toda su extensión, excepto a nivel de la vena cava inferior.

Para Branca (1914) el saco pericárdico es una membrana rígida que que tiene aspecto de una aponeurosis, descripción semejante a la citada por Goss (1977) donde Branca añade que alcanza un espesor de 400 micras, a diferencia de nuestra opinión donde el pericardio es una membrana flexible y de 0,26 mm de espesor. Por su parte, Latremouille et $a l$. señalaron que el espesor del pericardio gradualmente se incrementaba de 0,1 a 0,6 mm, alcanzando su máximo a nivel del diafragma, a lo largo del lado izquierdo del corazón. El tipo de tejido que encontramos interpuesto entre el pericardio y el músculo diafragma corresponde a tejido conjuntivo laxo, concordando con las descripciones de Gérard, Fusari, Rouvière (1959), Testut \& Latarjet y Williams et al., entre otros. Por su parte, Costacurta (1969) ha descrito ese tejido como fibro-elástico. Refiriéndose a la unión propiamente tal entre ambas estructuras, ésta se realiza a través de entrecruzamiento de fibras, disposición expresada por Sappey (1876), Pereira-Guimaraes (1894) y Falcone (1931), mientras que Poirier \& Charpy acrecentaron que ocurre en el margen anterior.

Nuestros resultados nos permiten afirmar que el entrecruzamiento de fibras ocurre principalmente a nivel de la parte central y en la hojuela derecha del tendón central. La separación de las estructuras en cuestión es fácil en los lugares donde existe el tejido conjuntivo laxo, pero los entrecruzamientos de fibras y la existencia del tejido que conforma los ligamentos freno-pericárdicos dificulta la separación en el margen anterior del bloque pericardio diafragmático, parte central y hojuela derecha, concordante con las aseveraciones de Paturet, Schaeffer (1942) y Testut \& Latarjet.

Lo descrito por Falcone de que el intercambio de fibras tendinosas es tan íntimo que imposibilita la separación de las dos estructuras no corresponde a lo que visualizamos al disecar nuestras piezas, ya que tales elementos son separables en su parte posterior, situación ya comentada por Versari y Testut \& Latarjet.

Entre los autores que describieron la constitución del pericardio y su relación con el diafragma, Wallraff; Schaeffer; DiDio y Braunwald (1988) señalaron que fibras colágenas que conforman el saco pericárdico están entrelazadas y Popa \& Lucinescu (1932); Debrünner; Gardner et al. (1967); Benninghoff \& Goerttler (1978) y Hollinshead (1980) indicaron que los ligamentos frenopericárdicos anterior y lateral son proyecciones de esa capa fibrosa. Con tales detalles morfológicos coincidimos plenamente.

Los resultados obtenidos en este trabajo complementarán los conocimientos morfológicos sobre el pericardio fibroso y sus relaciones con el diafragma.

BINVIGNAT, O. \& OLAVE, E. Biometric and morphological charachteristics of the fibrous pericardium and the diaphragm in Man Int. J. Morphol., 27(3):841-847, 2009.

SUMMARY: The pericardium is a fibrous and serous membrane that surround the heart and the juxta- cardiac portion of the great vessels. We studied the pericardium and diaphragm and we recorded different measurements, relations and connection between both. We dissected 142 mediastinal regions from $10 \%$ formaldehyde - fixed or fresh individual cadavers, Brazilian adults, of both sexes, from 18-70 years of age. For the histology study from both structures were sectioned five fragments of different regions: anterior, next to sternum (region 1), left lateral, next to heart apex (region 2), posterior (region 3), right lateral, next to course of inferior vena cava (region 4) and central(region 5). The average of transversal and anterior-posterior diameters of pericardium were $103.3+6.7 \mathrm{~mm}$ and $66.0 \pm 2.3$ $\mathrm{mm}$, respectively; the same diameters of diaphragm were $309.4 \pm 27.4 \mathrm{~mm}$ and $152.5 \pm 24.9 \mathrm{~mm}$, respectively. The diaphragm area was $37,260 \pm 2,324 \mathrm{~mm}^{2}$ and the area of pericardium base over the diaphragm was $6,042 \pm 367 \mathrm{~mm}^{2}$. The thickness of diaphragm was 2.42 $\pm 0.34 \mathrm{~mm}$ in right part, $2.38 \pm 0.71 \mathrm{~mm}$ in left part and $2.52 \pm 0.66 \mathrm{~mm}$ in anterior part. The thickness of pericardium was $0.26 \pm 0.02$ $\mathrm{mm}$. In region 2 both structures were easily separated in $47.2 \mathrm{~mm}$; in the region 4 both structures are fused. The results of this study will complement the morphologic knowledges about fibrous pericardium and its relationships with the diaphragm.

KEY WORDS: Anatomy; Diaphragm; Morphology; Biometry. 


\section{REFERENCIAS BIBLIOGRÁFICAS}

Abbas, A.E.; Appleton, C.P.; liu, P.T. \& Sweeney, J.P. Congenital absence of the pericardium: case presentation and review of literature. Int. J. Cardiol., 98 (1):21-5, 2005.

Anile, A. L'anatomia sistemática del Uomo. Napoli, Elpis, 1919.

Basmajian, J. V. Anatomía. 7. ed. México, Interamericana, 1980.

Benninghoff, A. \& Goerttler, K. Trattato di anatomia umana funzionale. Padova, Piccin, 1978.

Berdal, H. Noveaux éléments d'histoloqie normale. 6. ed. Paris, A. Maloine, 1906.

Bouchet, A. \& Cuilleret, J. Anatomía descriptiva, topográfica y funcional. México, Panamericana, 1979.

Branca, A. Précis d' histologie. 3. ed. Paris, Baillière, 1914.

Braunwald, E. Heart disseasse. 3. ed. Philadelphia, W. B. Saunders Company, 1988.

Bruce, J. ; Walmsley, R. \& Ross, J. A. Manual of surgical anatomy. London, E.\&S. Livingstone, 1964.

Chiarugi, G. Istituzioni di anatomia del Uomo. 2. ed. Milano, Libraria, 1924.

Costacurta, L. Histologia. São Paulo. Artes Médicas Ltda, 1969.

Davis,G. Anatomia applicata. Milano, Libraria, 1913.

Debrünner, W. Estrutura e função do pericárdio humano. Anatomischer Anzeiger 119 (6): 525-37,1956.

DiDio, L. J. A. Anatomia aplicada do pericárdio. Arquivos Brasileiros de Cardiologia. 44 (6):373-6, 1985.

Falcone, C. Trattato di anatomia umana. Milano, Vallardi, 1931.

Fort, J. A. Anatomie descriptive et dissection. Paris, Vigot Frères, 1902.

Fumagalli, Z. \& Cavallotti, C. Anatomia umana normale. Padova, Piccin, 1982.
Fusari, R. Compêndio di anatomia umana. Torino, Unione Tipografico-Éditrice Torinese, 1913.

Gardner, E.; Gray, D. J. \& O'Rahilly, R. Anatomia. 4. ed. Rio de Janeiro, 1967.

Gerard, G. Manuel d'anatomie humaine. Paris, G. Steinheil, 1912.

Goss, C. M. Gray anatomia. 2. ed. Rio de Janeiro, Guanabara Koogan 1977.

Hollinshead, W. H. Livro texto de anatomia humana. São Paulo, Harbra Harper \& Row do Brasil, 1980.

Landouzy, L. \& Bernard, L. Éléments dánatomie et de physilogie médicales. Paris, Masson et Cie., 1921.

Latremouille, C.; Vincentelli, A.; Zegdi, R.; D'Atellis, N.; Chachques, J.C.; Lassau, J.P.; Fabiani, J.N. \& Carpentier, A. The pericardium: a heterogeneous tissue. Anatomic and morphometric considerations. Morphologie, 83(260):41-4, 1999.

Leeson, C.R.\& Leeson, T. S. Anatomía Humana. México, Interamericana, 1972.

Le Gros Clark, W. E. Practical anatomy. 2. ed. London, Edward Amold \& Co., 1949.

Lochhart, R. D. ; Hamilton, G. F. \& Fyfe, F. W. Anatomy of the human body. London, Faber and Faber Limited, 1972.

Maisonnet, J. \& Coudane, R. Anatomie clinique et opératoire. Paris, G. Doin \& Cie., 1950.

Moore, K. L. Anatomia orientada para a clínica. 2. ed. Rio de Janeiro, Guanabara, 1990.

Orts Llorca, F. Anatomía humana. 4. ed. Madrid, Científico Médica, 1972.

Paturet, G. Traité d'anatomie humaine. Paris, Masson \& Cie., 1958.

Pereira-Guimaraes, J. Tratado de anatomia descriptiva. Rio de Janeiro, H. Laemmert \& Cie, 1894.

Poirier, P. \& Charpy, A. Traité d'anatomie humaine. 2. ed. Paris, Masson et Cie, 1902. 
Popa, G. T. \& Lucinescu, E. The mechanostructure of the pericardium. Journal of Anatomy. 67(1):78-107, 1932.

Prives, M.; Lisenkov, N. \& Bushkovich, V. Anatomia humana. 5a ed. Moscu, MIR, 1984.

Romanes, G. J. Cunninghan's manual de anatomia prática. São Paulo, Atheneu, 1976.

Rouvière, H. Anatomie humaine. 8. ed. Paris, Masson, 1959.

Sappey, PH. C. Traité d'anatomie descriptive. Paris, V. Adrien Delahaye et Cie, 1876.

Scapellato, F.; Imparato A.; Brunetto, A.; Temporelli, P.L. \& Giannuzzi, P. A paradigmatic case of partial agenesis of the pericardium. G. Italian Cardiol.,26(10):1187-93, 1996.

Schaeffer, J. P. "Morris" human anatomy. 10. ed. Philadelphia. The Blakiston Company, 1942.

Sharma, O.P. Pericardio-diaphragmatic rupture: five new cases and literature review. J. Emerg. Med., 17(6):9638, 1999.

Snell, R. S. Anatomia. 2. ed. Rio de Janeiro, Medsi, 1981.

Tandler, J. Tratado de anatomía sistemática. Barcelona, Salvat S. A., 1928.

Testut, L. \& Latarjet, A. Tratado de anatomía humana. 9. ed. Barcelona, Salvat, 1954.

Tillaux, P. Traité d'anatomie topographique avec applications a la chirurgie. 11. ed. Paris, Asselin et Houzeau, 1908.

Valenti, G. Compêndio di anatomia de H'uomo. Milano, Vallardi, 1909.

Versari, R. in Balli, Bertelli. Trattato di anatomia umana. 2. ed. Milano, Vallardi, 1932.

Wallraff, J. Uber den Bau und die kreisauffunktionen des Menschlichen Herbeutels. Klinische Wochenschrift. 16; (48):1665-9,1937.

Williams, P.; Warwick, R. ; Dyson, M \& Bannister, L. Gray Anatomia. 37. ed. Rio de Janeiro, Guanabara-Koogan, 1995.
Dirección para correspondencia:

Prof. Dr. Octavio Binvignat Gutiérrez

Disciplina de Anatomía Humana

Facultad de Ciencias de La Salud

Universidad Autónoma de Chile

Talca

CHILE

Email: obinvignatg@uautonoma.cl binvigna@terra.com.br

Recibido : 17-05-2009

Aceptado: 26-06-2009 
